\title{
A Method for the Spectroscopic Diagnostic of the Peculiarities of Materials Using the Semi-Auger Effect
}

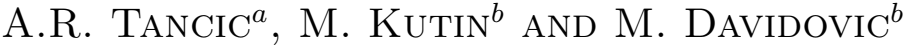 \\ ${ }^{a}$ VINCA Institute for Nuclear Sciences, P.O. Box 522, 11001 Belgrade, Serbia \\ ${ }^{b}$ Institute GOSA for Research and Development \\ Milana Rakića 35, 11000 Belgrade, Serbia
}

\begin{abstract}
We investigated the low energy satellites in the Röntgen spectra using the many-particle theory. These satellites are results of the radiative semi-Auger decay of the inner vacancy states. We did not treat only ordinary radiative decay. There exist the excitations of one of the atomic electrons together with the photon emission. We presented results of radiative semi-Auger decays of vacancy states in Ar atom. The knowledge of these processes is important for understanding the same processes in the case of the nanoparticles (molecules, fullerenes, clusters). Research in the field of the new materials requires more sophisticating methods which will open the new possibilities for obtaining materials of particular design, too. The theory which is presented in the paper may be easily generalized in case of particles like fullerenes and clusters.
\end{abstract}

PACS numbers: 32.30.Rj, 32.80.Wr

\section{Introduction}

The building of new materials in the field of the nanostructure and of nanotubes comes from a need of the theoreticians and experimentalists for more sophisticated methods and investigations for new possibilities for obtaining materials of particular design. According to that, we demonstrated in this paper the appearance of new structural effects which basically defined the diagnostic of material features by the different methods like the Röntgen analysis of materials, the Auger spectroscopy (for example, new compounds which are important in the theory of the superconductivity, of the magnetic materials, etc).

In our paper we observed, from the first principles, the appearance of new satellite lines in the Röntgen atomic spectra, and correspondently, in the macromolecules, clusters, fullerenes, etc., too. The appearance of the new lines is connected with the process of radiative semi-Auger (RSA) decay of the inner shell vacancy. The electron transition from the outher subshell to the hole state is evaluated together with the discrete excitation of an atomic electron. Recently, there exist three methods of the calculations of such processes:

1. The configuration interaction method (CI) [1].

2. The Cooper-Aberg method (the "shake" method) (CA) $[2,3]$.

3. The many-body theory (MT), the Hartree-Fock approximation (HF), the many-body perturbation theory (PT), and random phase approximation with exchange (RPAE) (which is exactly the special kind of treating the perturbation theory [4-6]). In this paper we use the RPAE method $[7,8]$.
The methods 1 and 2 take into account only one of the possible decay mechanisms of RSA decay. The method which we used takes into account not only the processes which are described by the methods 1 and 2, but also other important processes. Many of the satellite lines are obtained experimentally, too $[3,9]$.

As an example, we presented our calculations for the Ar atom. We obtained three groups of satellites.

\section{Theory}

The probability of a process in the framework of the dipole approximation is determined $[6,8]$ as

$$
\begin{aligned}
W_{\mathrm{i}} & =\frac{\alpha^{3} \omega^{3}}{[L][S]} \sum_{M_{L} M_{L^{\prime}} M_{S} M_{S^{\prime}}}|M|^{2}, \\
M & =\left\langle\Psi_{\mathrm{f}}\left(L^{\prime} S^{\prime} M_{L^{\prime}} M_{S^{\prime}}\right)|(\boldsymbol{d} \cdot \boldsymbol{e})| \Psi_{\mathrm{i}}\left(L S M_{L} M_{S}\right)\right\rangle .
\end{aligned}
$$

$M$ is the amplitude of a radiative transition, $\Psi_{\mathrm{i}, \mathrm{f}}$ are the wave functions of the initial and final state of the atom with the one hole and two vacancies $j_{1}, j_{2}$ and one electron $g$ in the discrete level, $\alpha$ is the fine structure constant, $\boldsymbol{e}$ is the polarization vector of the emitted quantum, $\boldsymbol{d}=\sum_{i} \boldsymbol{r}_{i}$ is the operator of the atomic dipole moment, $L, S$ are the orbital and spin momenta, and $M_{L}$, $M_{S}$ are their projections. The probability (1) has the dimension of energy and is expressed in rydbergs.

In the calculation of the decay amplitude the PT usually is applied, since the energy gap between atomic subshells is rather large, and the matrix elements of the Coulomb interaction between the electrons in different subshells are relatively small. We use the many-particle PT, exactly the RPAE method $[5,6,10]$. In the lowest order in the electron interaction the amplitude of presented decay is determined by the diagrams in Fig. 1: a 
line with a right (left) arrow denotes the one-electronic (-hole) state; a line with a double arrow to the right denotes the discrete excited state; the dashed line - photon and the wavy line represents the Coulomb interaction. The summation over all momenta is implied in (1). The total amplitude is the sum of the contributions of the presented diagrams $(A, B, C, D)$ in Fig. 1. For example (diagram $A)$ :

$$
M_{1}=\sum_{\Pi} F \sum_{k}\langle\mathrm{i}|(\boldsymbol{r} \cdot \boldsymbol{e})| k\rangle\left\langle k g|U| j_{1} j_{2}\right\rangle E_{k g j_{1} j_{2}}^{-1} .
$$

In Eqs. (2) (and corresponding expressions for $M_{2,3,4}$ ), $E_{k l m n}=E_{k}+E_{l}-E_{m}+E_{n}$, where $E_{\nu}$ are the HF energies, and, as usual, the matrix $U$ present the difference between the direct and exchange matrix elements. The summation $\sum_{\Pi}$ includes all the projections of orbital and spin momenta of initial and final vacancies, as well as the factor $F$ is determined as [10-12]:

$$
\begin{aligned}
F & \sim C_{l_{\mathrm{f}_{1}} m_{\mathrm{f}_{1}} l_{\mathrm{f}_{2}} m_{\mathrm{f}_{2}}}^{L_{1} M_{L_{1} M_{L_{1}} l_{g} m_{g}}} C_{1 / 2 \mu_{\mathrm{f}_{1}} 1 / 2 \mu_{\mathrm{f}_{2}}}^{L^{\prime} M_{L^{\prime}}} C_{S_{1}}^{S_{1}} \\
& \times C_{S_{1} M_{S_{1}} 1 / 2 \mu_{\mathrm{f}_{g}}}^{S^{\prime} M_{S^{\prime}}}(-1)^{l_{\mathrm{i}}+l_{\mathrm{f}_{1}}+l_{\mathrm{f}_{2}}+3 / 2-m_{\mathrm{i}}-\mu_{\mathrm{i}}-m_{\mathrm{f}_{1}}-\mu_{\mathrm{f}_{1}}} \\
& \times x(-1)^{-m_{\mathrm{f}_{2}}-\mu_{\mathrm{f}_{2}}} .
\end{aligned}
$$

Here $C$ presents the Clebsh-Gordan coefficients. The factor $F$ and summation over the projection in (2) allow that the angular momenta of the holes of the in and out states are linked into definite term $L S\left(L^{\prime} S^{\prime}\right)$ [13].
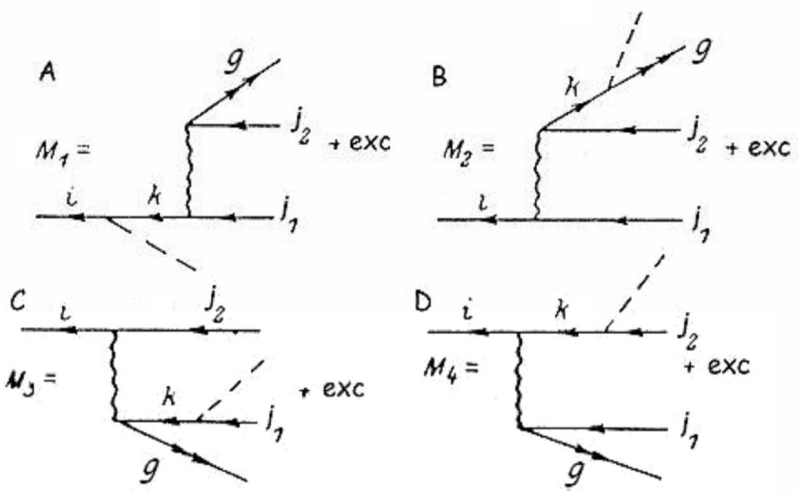

Fig. 1. The amplitude of the semi-Auger radiative decay - RSA; the exchange parts of diagrams are denoted by "exc".

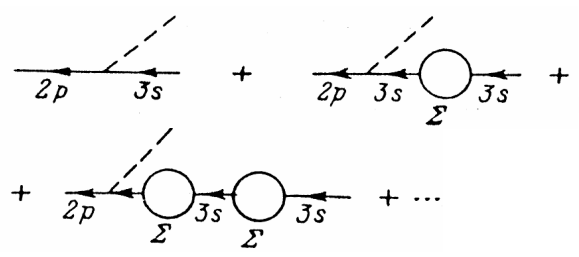

Fig. 2. The influence of the $3 s$-vacancy renormalization to the probability of RSA of the state $2 p^{-1}$.
After integration of the angle and spin variables and summation of the orbital and spin momenta projections of the holes we obtain [7] a very complex final expression which contains all known mechanisms of RSA decay (including processes which are described by methods 1 and 2).

If in the initial state the influence of the other configurations is very small, the RSA amplitude (which takes into account the mixing of configurations in the final state) may be given as in Fig. 2 .

Here $\Sigma$ is the diagonal matrix element of the self-energy part of the one-hole Green function

$$
\begin{aligned}
& G_{k_{1}}: \Sigma_{k_{1}}(\varepsilon)= \\
& \quad \sum_{p}\left|\left\langle k_{1} p|V| \mathrm{f}_{1} \mathrm{f}_{2}\right\rangle\right|^{2} /\left(\varepsilon+E_{p}-E_{\mathrm{f}_{1}}-E_{\mathrm{f}_{2}}\right) .
\end{aligned}
$$

We calculated this quantity in the improved version of the RPAE method by using the contributions of some important third-order diagrams $[6,12,14]$. After the summation of the diagrams in Fig. 2, the following expression is obtained:

$$
M=\left\langle k_{1}|(\boldsymbol{r} \cdot \boldsymbol{e})| \mathrm{i}\right\rangle\left[1-\Sigma_{k_{1}}(\varepsilon) /\left(\varepsilon-E_{k_{1}}-\mathrm{i} \delta\right)\right] .
$$

The final expression for the probability of the decay of the vacancy $\mathrm{i}$ is $[5,6]$ :

$$
\begin{aligned}
& \frac{\mathrm{d}}{\mathrm{d} \omega} W_{\mathrm{i}}=W_{\mathrm{i}}^{\mathrm{HF}} \sum_{p=0}^{\infty} F_{p} \delta\left(\varepsilon-\varepsilon_{p}\right), \\
& F_{p}=\left[1-\left(\partial E_{k_{1}}(\varepsilon) / \partial \varepsilon\right)_{\varepsilon=\varepsilon_{p}}\right]^{-1} .
\end{aligned}
$$

$F_{p}$ is the spectroscopic factor, $\varepsilon_{p}$ values are obtained from the equation $\varepsilon-E_{k_{1}}-E_{k_{1}}(\varepsilon)=0$.

\section{Results of the calculations and conclusions}

In this work we calculated the probabilities of the RSA of the $2 p$ vacancy in Ar. We obtained that except the $2 p^{-1} \rightarrow 3 p^{-2}\left[{ }^{1} D\right] 3 d, 4 d\left[{ }^{2} S\right]$ satellites, there appear the lines which are connected with the transitions in $3 p^{2}\left[{ }^{1} S,{ }^{3} P\right] x n d\left[{ }^{2} P,{ }^{2} D\right]$ states, too. The calculations demonstrate that the strongest transition is $2 p^{-1} \rightarrow$ $3 p^{-2}\left[{ }^{3} P\right] 3 d\left[{ }^{2} D\right]$. The probability of this transition is only 2.5 times smaller that the probability of the main satellite $2 p^{-1} \rightarrow 3 p^{-2}\left[{ }^{1} D\right] 3 d, 3 d\left[{ }^{2} S\right]$.

\subsection{Decay of the $2 p$ vacancy in $A r$}

The satellite spectra in Ar is more complex that the corresponding spectra in the case of the $s$-vacancy decays. In Table I we presented the results of the energies and probabilities which are obtained in the RSA decay of the $2 p$ vacancy. According to the selection rules, in the case of the $p$ hole decays, the orbital momentum of the final state has the possible values: $L^{\prime}=0,1,2$. Like in the case of $\mathrm{Ne}[5,6]$ here we have three groups of satellites, see Table II. The distance between groups is $\approx 1-2 \mathrm{Ry}$. 
TABLE I

Energies and probabilities of the RSA transition in Ar. The decay of the $2 p$ vacancy.

\begin{tabular}{l|c|c|c}
\hline \hline \multicolumn{1}{c|}{ Transition } & $\begin{array}{c}\text { Photon energy } \\
{[\mathrm{Ry}]}\end{array}$ & $\begin{array}{c}\text { Probability } \\
\text { (Ref. }[5]) \\
{\left[10^{-7} \mathrm{eV}\right]}\end{array}$ & $\begin{array}{c}\text { Probability } \\
\text { (this work) } \\
{\left[10^{-7} \mathrm{eV}\right]}\end{array}$ \\
\hline $2 p^{-1}\left[{ }^{2} P\right] \rightarrow 3 s^{-2}\left[{ }^{1} S\right] 4 s\left[{ }^{2} S\right]$ & 13.6 & 1.64 & 1.70 \\
$2 p^{-1}\left[{ }^{2} P\right] \rightarrow 3 s^{-1} 3 p^{-1}\left[{ }^{1} P\right] 4 p\left[{ }^{2} S\right]$ & 15.3 & 0.26 & 0.28 \\
$2 p^{-1}\left[{ }^{2} P\right] \rightarrow 3 s^{-1} 3 p^{-1}\left[{ }^{1} P\right] 5 p\left[{ }^{2} S\right]$ & 13.0 & 0.21 & 0.24 \\
$2 p^{-1}\left[{ }^{2} P\right] \rightarrow 3 s^{-1} 3 p^{-1}\left[{ }^{3} P\right] 4 p\left[{ }^{2} S\right]$ & 14.7 & 3.93 & 4.01 \\
$2 p^{-1}\left[{ }^{2} P\right] \rightarrow 3 s^{-1} 3 p^{-1}\left[{ }^{3} P\right] 5 p\left[{ }^{2} S\right]$ & 14.4 & 0.17 & 0.19 \\
$2 p^{-1}\left[{ }^{2} P\right] \rightarrow 3 p^{-2}\left[{ }^{1} S\right] 4 s\left[{ }^{2} S\right]$ & 15.5 & 0.10 & 0.13 \\
$2 p^{-1}\left[{ }^{2} P\right] \rightarrow 3 p^{-2}\left[{ }^{1} S\right] 3 d\left[{ }^{2} D\right]$ & 15.4 & 10.21 & 10.22 \\
$2 p^{-1}\left[{ }^{2} P\right] \rightarrow 3 p^{-2}\left[{ }^{3} P\right] 3 d\left[{ }^{2} P\right]$ & 15.8 & 0.19 & 0.23 \\
$2 p^{-1}\left[{ }^{2} P\right] \rightarrow 3 p^{-2}\left[{ }^{3} P\right] 3 d\left[{ }^{2} D\right]$ & 15.8 & 24.16 & 25.04 \\
$2 p^{-1}\left[{ }^{2} P\right] \rightarrow 3 p^{-2}\left[{ }^{1} D\right] 3 d\left[{ }^{2} S\right]$ & 15.7 & 64.50 & 66.35 \\
$2 p^{-1}\left[{ }^{2} P\right] \rightarrow 3 p^{-2}\left[{ }^{1} D\right] 4 d\left[{ }^{2} S\right]$ & 15.2 & 27.62 & 29.41 \\
$2 p^{-1}\left[{ }^{2} P\right] \rightarrow 3 p^{-2}\left[{ }^{1} D\right] 3 d\left[{ }^{2} P\right]$ & 15.7 & 0.28 & 0.31 \\
$2 p^{-1}\left[{ }^{2} P\right] \rightarrow 3 p^{-2}\left[{ }^{1} D\right] 3 d\left[{ }^{2} D\right]$ & 15.7 & 12.55 & 12.78
\end{tabular}

TABLE III

Energies and probabilities of the RSA transition in Ne. The decay of the $1 s$ vacancy.

\begin{tabular}{l|c|c|c}
\hline \hline \multicolumn{1}{c|}{ Transition } & $\begin{array}{c}\text { Photon energy } \\
{[\mathrm{Ry}]}\end{array}$ & $\begin{array}{c}\text { Probability } \\
(\text { Ref. [5]) } \\
{\left[10^{-5} \mathrm{eV}\right]}\end{array}$ & $\begin{array}{c}\text { Probability } \\
\text { (this work) } \\
{\left[10^{-5} \mathrm{eV}\right]}\end{array}$ \\
\hline $1 s^{-1}\left[{ }^{2} S\right] \rightarrow 2 s^{-2}\left[{ }^{1} S\right] 3 p\left[{ }^{3} P\right]$ & 55.1 & 0.03 & 0.04 \\
$1 s^{-1}\left[{ }^{2} S\right] \rightarrow 2 s^{-1} 2 p^{-1}\left[{ }^{1} P\right] 3 s\left[{ }^{2} P\right]$ & 57.6 & 1.28 & 1.23 \\
$1 s^{-1}\left[{ }^{2} S\right] \rightarrow 2 s^{-1} 2 p^{-1}\left[{ }^{3} P\right] 3 s\left[{ }^{3} P\right]$ & 58.4 & 3.13 & 3.21 \\
$1 s^{-1}\left[{ }^{2} S\right] \rightarrow 2 s^{-1} 2 p^{-1}\left[{ }^{1} P\right] 3 d\left[{ }^{2} P\right]$ & 57.0 & 0.10 & 0.15 \\
$1 s^{-1}\left[{ }^{2} S\right] \rightarrow 2 s^{-1} 2 p^{-1}\left[{ }^{3} P\right] 3 d\left[{ }^{2} P\right]$ & 57.8 & 0.06 & 0.07 \\
$1 s^{-1}\left[{ }^{2} S\right] \rightarrow 2 p^{-2}\left[{ }^{1} S\right] 3 p\left[{ }^{2} P\right]$ & 59.5 & 4.12 & 3.98 \\
$1 s^{-1}\left[{ }^{2} S\right] \rightarrow 2 p^{-2}\left[{ }^{1} S\right] 4 p\left[{ }^{2} P\right]$ & 59.1 & 0.52 & 0.31 \\
$1 s^{-1}\left[{ }^{2} S\right] \rightarrow 2 p^{-2}\left[{ }^{3} P\right] 3 p\left[{ }^{2} P\right]$ & 60.0 & 39.29 & 41.82 \\
$1 s^{-1}\left[{ }^{2} S\right] \rightarrow 2 p^{-2}\left[{ }^{3} P\right] 4 p\left[{ }^{2} P\right]$ & 59.6 & 5.38 & 6.22 \\
$1 s^{-1}\left[{ }^{2} S\right] \rightarrow 2 p^{-2}\left[{ }^{1} D\right] 3 p\left[{ }^{2} P\right]$ & 59.9 & 30.07 & 33.32 \\
$1 s^{-1}\left[{ }^{2} S\right] \rightarrow 2{ }^{-2}\left[{ }^{1} D\right] 4 p\left[{ }^{2} P\right]$ & 59.5 & 4.25 & 4.29
\end{tabular}

In our calculations we use, except the diagrams in Fig. 2, also the diagrams in Fig. 3 which take into account the contributions of the virtual excitations in the RSA process. Then in formula (4) we must change $W_{\mathrm{i}}^{\mathrm{HF}} \rightarrow W_{\mathrm{i}}^{\text {corr }}$.

In Table III we give our earlier obtained results of the [6-8] RSA method (for comparison). In this case three groups of the satellites arise, too. The mean distance between the satellites is approximately $2 \mathrm{Ry}$.
The probabilities of the RSA decay in the general case depend: firstly, on the electronic configuration in the final state configuration, and secondly, inside each of group of satellites depend on the residual-ion term, which is defined by the quantity $F^{2}$ (the quantity $F \sim$ $\left.\left(2 L^{\prime}+1\right)\left(2 S_{1}+1\right)\left(2 L_{1}+1\right)\right)$ and angular and spin terms in the partial amplitudes. 
TABLE II

RSA decay of the $2 p$ vacancy in Ar. Three groups of satellites are obtained.

\begin{tabular}{l|l|l}
\hline \hline 1. & $2 p^{-1} \rightarrow$ & $3 s^{-2} n s\left[{ }^{2} S\right]$ or $n d\left[{ }^{2} D\right]$ \\
\hline 2. & $2 p^{-1} \rightarrow$ & $3 s^{-1} 3 p^{-1}\left[{ }^{1,3} P\right] n p\left[{ }^{2} S,{ }^{2} P,{ }^{2} D\right]$ or $n f\left[{ }^{2} D\right]$ \\
\hline & & $3 p^{-2}\left[{ }^{1} S,{ }^{3} P,{ }^{1} D\right] n s\left[{ }^{2} S,{ }^{2} P,{ }^{2} D\right]$ \\
3. & $2 p^{-1} \rightarrow$ & $3 p^{-2}\left[{ }^{1} S,{ }^{3} P,{ }^{1} D\right] n d\left[{ }^{2} S,{ }^{2} P,{ }^{2} D\right]$ \\
& & $3 p^{-2}\left[{ }^{1} S,{ }^{3} P,{ }^{1} D\right] n g\left[{ }^{2} S,{ }^{2} P,{ }^{2} D\right]$
\end{tabular}
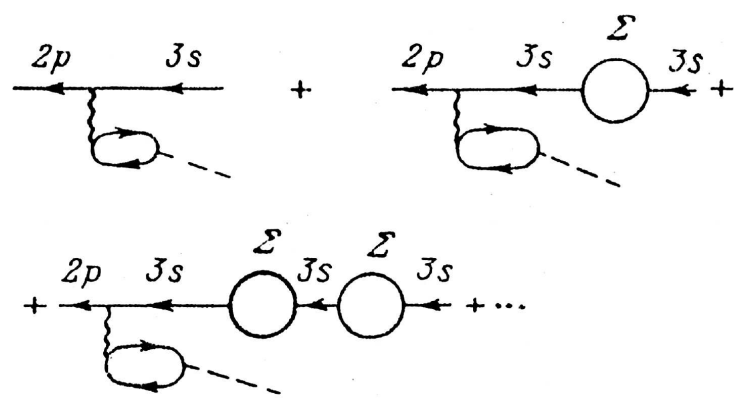

Fig. 3. The contributions of the virtual excitation processes in the decay of the $2 p$-vacancy.

\subsection{Conclusion}

This paper presents the method of calculations of radiative semi-Auger decay in the framework of the many-body theory. We demonstrated the possibility to find the correct positions of defined lines of spectra of Ar atom.

The method which we presented here may be applied on other noble gases atoms, and also on atoms (molecules) with great polarizability (for example, on the atoms of alkali metals). Also, using the theoretical generalization in the calculations which are connected with the clusters and fullerenes, and generalization of the polarizability, it is possible to calculate the energies and probabilities of the corresponding decays $[5,6,15,16]$ (for example, the effects of the semi-Auger decays) for the $\mathrm{C}_{60}, \mathrm{C}_{60}^{+}, \mathrm{Na}_{40}, \mathrm{Na}_{20}^{+}, \mathrm{Ag}_{11}^{+}$, i.e. to identify the spectra of the corresponding atoms, ions, fullerenes and clusters in material which we investigated. This is very important for obtaining the accurate diagnosis of some materials.
By that we open the possibility to design new materials in nano- and macro-domain, too.

\section{Acknowledgments}

We are very grateful to Prof. M.Ya. Amusia for fruitful discussion about this work and many helps.

The presented work is supported by the Ministry of Science and Technological Development of the Republic of Serbia (the projects No. 141001 and 141030G).

\section{References}

[1] K. Duall, F. Larkins, J. Phys. B 15, 1725 (1982).

[2] J.W. Cooper, R.E. La Willa, Phys. Rev. Lett. 25, 1745 (1970).

[3] T. Aberg, Phys. Rev. 4A, 1735 (1971).

[4] V. Bergamann, P. Glatze, J.H. Robblee, J. Messinger, C. Fernandez, R. Cinco, H. Visser, K. McFarlane, E. Bellacchio, S. Pizarro, K. Sauer, V. Yashandra, M.P. Klein, B.L. Cox, K.H. Nealson, S.P. Cramra, J. Synchrotron Radiat. 8, 199 (2001).

[5] M.Ya. Amusia, A. Kheifets, J. Phys. B 18, L679 (1985).

[6] A.R. Tancic, M. Davidovic, Mater. Sci. Forum 518, 331 (2006).

[7] A.R. Tancic, M. Davidovic, in: Yucomat 200\%, The Book of Abstracts, Institute of Technical Sciences, Serbian Academy of Sciences and Arts, Belgrade, p.112.

[8] M.Ya. Amusia, V. Kilin, A. Kolesnikova, I. Lee, Sov. J. Tech. Phys. Lett. 11, 343 (1985).

[9] T. Kurisaki, S. Matsuo, I. Toth, H. Wakita, Analyt. Sci. 24, 1385 (2008).

[10] G.F. Gribakin, J. Ludlow, Phys. Rev. Lett. $\mathbf{8 8}$, 163202 (2002).

[11] B. Akabayov, C.J. Doonen, J.I. Pickering, G.N. George, I. Sagi, J. Synchrotron Radiat. 12, 392 (2005).

[12] M. Amusia, A. Hajfec, Zh. Eksp. Teor. Fiz. 86, 1217 (1984).

[13] B.R. Judd, 1967, Second Quantisation and Atomic Spectroscopy, Hopkins, Baltimore 1967.

[14] A.R. Tancic, in: Int. Conf. on Theor. Phys. TH-2002, Book of Abstracts, Birkhauser, Paris 2003, p. 272.

[15] R. La Villa, Phys. Rev. A 4, 476 (1971).

[16] Kawai Jun, Analyt. Sci. 21, 733 (2005). 